\title{
Developmental hematopoiesis: ontogeny, genetic programming and conservation
}

Aldo Ciau-Uitz ${ }^{1}$, Rui Monteiro ${ }^{1,2}$, Arif Kirmizitas ${ }^{1}$ and Roger Patient ${ }^{1,2 \#}$

${ }^{1}$ MRC Molecular Haematology Unit, Weatherall Institute of Molecular Medicine, John Radcliffe Hospital, University of Oxford, Oxford, OX3 9DS, UK

${ }^{2}$ BHF Centre of Research Excellence, Oxford, UK

\# Corresponding Author: Professor Roger Patient PhD, MRC Molecular Haematology Unit, Weatherall Institute of Molecular Medicine, John Radcliffe Hospital, University of Oxford, Oxford, OX3 9DS, UK. Phone: +44 1865 222613, FAX: +44 1865 222501, Email: roger.patient@imm.ox.ac.uk

Table of Content Category: Stem Cell Biology

Genomics and Model Organisms: New Horizons for Experimental Hematology special issue.

Word Count $=3658$ 


\section{Abstract}

Hematopoietic stem cells (HSCs) sustain blood production through life and are of pivotal importance in regenerative medicine. Although HSC generation from pluripotent stem cells would resolve their shortage for clinical applications, this has not yet been achieved mainly due to the poor mechanistic understanding of their programing. Bone marrow HSCs are first created during embryogenesis in the dorsal aorta (DA) of the mid-gestation conceptus, from where they migrate to the fetal liver and, eventually, the bone marrow. It is currently accepted that HSCs emerge from specialised endothelium, the hemogenic endothelium, localised in the ventral wall of the DA through an evolutionarily conserved process called the endothelial to hematopoietic transition (EHT). However, EHT represents one of the last steps in HSC creation and an understanding of earlier events in the specification of their progenitors is required if we are to create them from naïve pluripotent cells. Due to their ready availability and external development, studies on zebrafish and Xenopus embryos have enormously facilitated our understanding of the early developmental processes leading to the programming of HSCs from nascent lateral plate mesoderm to hemogenic endothelium in the DA. The amenity of the Xenopus model to lineage tracing experiments has also contributed to the establishment of the distinct origins of embryonic (yolk sac) and adult (HSC) hematopoiesis, whilst the transparency of the zebrafish has allowed in vivo imaging of developing blood cells, particularly during and after the emergence of HSCs in the DA. Here, we discuss the key contributions of these model organisms to our understanding of developmental hematopoiesis.

Key words: Hematopoietic stem cell, Hemangioblast, Hemogenic endothelium, Development, Xenopus, Zebrafish. 


\section{Introduction}

HSCs residing in the bone marrow are the foundation of adult hematopoiesis and, through balanced self-renewal and differentiation, give rise to all mature blood cells through life. The bone marrow though, is seeded by HSCs generated during embryogenesis. How HSCs are first created in the embryo is a subject of intense research due to its potential translational applications such as the de novo generation of personalised HSCs for transplantation and other clinical purposes. The study of HSC ontogeny in mammalian embryos is made more difficult by their internal development. Critically, developmental hematopoiesis is an evolutionarily conserved process (Figure 1) and the same developmental principles apply to externally developing embryos, such as those of teleost fish and amphibians, allowing them to be used as models for hematopoiesis.

In the past few decades, the use of a variety of model organisms has resulted in numerous seminal discoveries and great progress in the field of developmental hematopoiesis. It has now been established that during embryogenesis blood cells are created in at least three waves[1] (Figure 1). The first and most familiar wave of embryonic hematopoiesis takes place in the yolk sac blood islands and equivalent tissues in externally developing embryos (Figure1, blue and green bars) and gives rise to a transient population of erythrocytes and primitive myeloid cells. The second wave, also deriving from the yolk sac but now derived from hemogenic endothelium embedded in the wall of functional blood vessels, gives rise to multipotent progenitor cells which migrate to the fetal liver (or its equivalent in the zebrafish, the CHT) and differentiate into erythro-myeloid and lymphoid lineages (Figure 1, pink bars). These two early waves do not generate bona fide HSCs capable of reconstitution of irradiated 
adult recipients. The third wave of blood creation (Figure 1, dark blue bars), initiates intraembryonically from hemogenic endothelium localised in the ventral wall of the DA and creates bona fide HSCs responsible for hematopoiesis in the late fetus and the adult bone marrow. During the course of these studies, new concepts, lineage relationships, tissue interactions and molecular players, as well as controversies, have emerged. In this review we briefly summarise some of these issues with an emphasis on the contributions of the zebrafish and Xenopus models.

\section{Embryonic hematopoiesis and the ontogeny of hematopoietic stem cells}

In mammalian and avian embryos, the first blood cells differentiate extra-embryonically in the yolk sac. Therefore, the yolk sac was initially considered to be the source of all blood cells in the embryo and the adult, particularly after the demonstration that the main embryonic hematopoietic organ, the liver, has no capacity for the de novo generation of blood cells but rather it is seeded, via the circulation, from an external source[2, 3]. Before the bone marrow, the main site of hematopoiesis in the adult, is seeded, the fetal liver, thymus and spleen function as hematopoietic organs[4]. The thymus and spleen form relatively late in gestation and are involved in the production of highly differentiated blood cells. However, the lack of hematopoiesis in organ cultures of both rudiments, isolated early in ontogeny, demonstrated that blood cells are not generated there in situ $[5,6]$. Furthermore, transplantation of thymus and spleen rudiments showed no donor derived hematopoiesis but rather that they are seeded from progenitors extrinsic to these organs $[7,8]$. The liver develops earlier in ontogeny, nevertheless, both rudiment cultures and transplantation experiments showed that it is also seeded by extrinsic hematopoietic progenitors. When the 
whole mouse hepatic primordium is taken before the 28 -somite stage and transplanted into chick or quail embryos, no donor derived hematopoiesis is observed even though normal hepatocyte differentiation takes place; nevertheless, same stage hepatic rudiments cultured in vitro become hematopoietic when supplied with exogenous HSCs[9]. Thus, because the yolk sac was at that time the only pre-liver hematopoietic organ, it was assumed that all blood cells, including HSCs, ultimately originate from it.

Evidence of the existence of an intra-embryonic source for hematopoietic cells was first provided by elegant inter-species quail-chick chimera experiments[10]. In these experiments, a quail embryo was engrafted onto a chick yolk sac (and vice versa) before the establishment of blood circulation between these compartments. Using this system, it was demonstrated that yolk sac blood progenitors are not endowed with self-renewing potential and that their progeny are gradually replaced by cells originating from the embryo proper, demonstrating the existence of self-renewing blood progenitors/stem cells of intra-embryonic origin[10, 11].

Further evidence supporting a non-yolk sac origin for blood stem cells was provided by chimeric Xenopus embryos. Transplanting tissues between triploid and diploid embryos or between two Xenopus sub-species, established that blood progenitors derive from two regions of the embryo, the ventral blood island (VBI) and the dorsal lateral plate (DLP) mesoderm $[12,13]$. Using this system, it was established that both VBI and DLP give rise to multipotent progenitors producing both erythrocytes and lymphocytes. Nevertheless, the VBI progeny were gradually replaced by the DLP progeny so that, after metamorphosis, virtually all blood cells were contributed by the DLP [12-14]. Importantly, it was also established that hepatic erythrocytes, leukocytes and B-cells in the liver, the main hematopoietic organ of Xenopus 
larvae, derive from the DLP [15]. Taken together, these experiments showed that blood cells derive from two regions of the early embryo, one equivalent to the extra-embryonic yolk sac, the VBI, and the other corresponding to the intra-embryonic source suggested by the chickquail chimeras, the DLP. The VBI produces large amounts of embryonic erythrocytes and a short burst of T-and B-cells in pre-metamorphosis larvae until the DLP/HSC progeny reach maturity $[13,15,16]$. In its turn, DLP derived stem cells seed the liver where they generate erythrocytes which replace those derived from the VBI and they also produce postmetamorphosis lymphocytes (Figure 1).

More recently, the intra-embryonic origin of stem/progenitor cells has also been confirmed in mouse and human embryos [17-21]. In vitro cultured mouse para-aortic splanchnopleura (PSp) explants taken before the onset of blood circulation are capable of producing multilineage progeny, including B-cells [19]. Importantly, CD34+ cells isolated from human P-Sp produce T- and B-cell progeny when transplanted into NOD-SCID mice [18]. However, more significantly, cells with HSC properties are first detected in the aorta, gonads and mesonephros (AGM) region of the E10.5 mouse embryo: only cells from the AGM have the capability of long-term reconstitution (LTR) when transplanted into adult mouse recipients $[17,22]$. Yolk sac cells do not exhibit LTR in adult recipients and a body of evidence indicates that they can only produce T- and B-cells [23-25]. Nonetheless, yolk sac, as well as P-Sp, derived T- and B-cells confirm the existence of the non-HSC derived lymphoid progenitors first discovered in Xenopus. Indeed, as in Xenopus, it has been suggested that yolk sac derived lymphopoiesis might be required before these lineages are generated by bona fide HSCs [23]. 
Lineage tracing has shown that definitive HSCs, rather than arising in intra-aortic clusters, form in the region between the DA and the axial vein and from there migrate to the caudal hematopoietic tissue (CHT) [26, 27]. Live imaging in CD41-GFP transgenic embryos showed that as HSCs emerge, they begin expressing low levels of CD41 (CD41-GFP ${ }^{\text {low }}$ cells) and initiate their migration to the CHT [27]. The CHT also serves as an intermediate niche for HSC expansion and the differentiation of erythroid and myeloid cells [26, 28, 29], indicating that the $\mathrm{CHT}$ is the teleost equivalent of the mammalian fetal liver (Figure 1). Although the zebrafish fetal liver and bone marrow are not hematopoietic, equivalent organs have been described. HSCs expand in the CHT and migrate to seed the thymus and kidney marrow [27, 30]. Furthermore, it has been shown that the kidney marrow contain cells capable of LTR of all blood lineages when engrafted into bloodless mutants or irradiated adult recipients [31, 32]. More recently, it was demonstrated that only the CD41-GFP low cells in the adult kidney marrow are capable of multilineage reconstitution [33]. Thus, the kidney marrow is the adult site of hematopoiesis in teleosts (Figure 1) and harbours bona fide CD41-GFP ${ }^{\text {low }}$ HSCs.

Transplantation experiments in Xenopus embryos demonstrated that at least three waves of non-HSC derived lymphoid progenitors seed both the liver and the thymus [14, 16, 34], and showed the existence of non-HSC derived erythrocytes that persist up to metamorphosis [35]. These observations suggested the generation of several types of non-HSC derived progenitors which served the larvae at different times of development but the ontogenic relationships between these progenitors remain unknown. Studies in the mouse have also revealed that after the yolk sac produces the wave of transient primitive cells, erythro-myeloid progenitors (EMPs) are produced $[36,37]$. These EMPs migrate to the embryo proper and seed the liver to produce fetal definitive erythrocytes and their yolk sac provenance has been demonstrated 
in mouse models lacking a functional circulation or heartbeat, VE-Cadherin-null [38] and NCX1-null [39] embryos, respectively, where few EMPs are found in the embryo proper. A similar wave of EMPs is produced in the posterior blood island of the zebrafish and, similarly, these EMPs are established before HSC emergence [40]. Regarding T- and B-cells, analysis of NCX1-null mouse embryos has demonstrated that lymphocyte progenitors are independently produced in the yolk sac and that they are capable of differentiating into innate B-1 type cells upon transplantation [24]. Transplantation of GFP-transgenic yolk sac onto wild type yolk sac has also indicated that the capacity to produce CD19+ B-cells does exist in the yolk sac [41]. Importantly, a lympho-myeloid progenitor cell has recently been isolated from the yolk sac before the emergence of HSCs in the AGM [23]. LinKit+Rag1GFP+IL7R $\alpha+$ progenitors possessing GM, B and T cell, but not MkE potential, are first detected in the E9.5 yolk sac when they already exhibit a combined lymphoid GM but not MkE transcriptional priming [23]. Taken together, the yolk sac produces at least two types of non-HSC derived multipotent progenitor cells in addition to primitive erythrocytes. Although their contribution to fetal and adult hematopoiesis is unclear, new hematopoietic tissues such as the head vasculature [42] and hemogenic endocardium [43], have recently been discovered in the embryo proper, indicating that our knowledge of developmental hematopoiesis is far from complete. Nevertheless, it is currently believed that at least five waves of unrelated hematopoietic progenitors are independently produced during mouse/mammalian embryonic development and that only the AGM produces bona fide HSCs [44].

Although the yolk sac does not produce progenitor cells endowed with LTR potential when transplanted into adult irradiated mice, yolk sac derived progenitors appear to acquire this 
potential when transplanted into the fetal or neonatal liver [24, 45, 46], suggesting that the yolk sac environment cannot provide an appropriate niche for HSC programing. This is supported by the fact that Xenopus VBI explants can produce HSC progeny when transplanted into the DLP environment and that DLP explants lose their HSC potential when transplanted into the VBI [47]. In addition, it has recently been shown that pre-HSCs are present within extra-embryonic arteries, not veins, and that these cells can be matured into HSCs by exposure to exogenous growth factors produced in the AGM niche but not in extraembryonic niches, such as interleukin 3 [48]. Controversially, it has been suggested that yolk sac blood progenitors migrate to the embryo proper to undergo further programming in the AGM environment to become HSCs [49]. However, this is unlikely since in chick-quail chimeras no contribution of the yolk sac to intra-embryonic blood was found [10]. Also, lineage labeling in Xenopus demonstrated distinct origins for the extra- and intra-embryonic lineages: even as early as the 32-cell stage these lineages do not share ancestry [50]. This argues that the niche plays an essential role in HSC programing and that the in vitro creation of HSCs requires an understanding of the intra-embryonic niche(s) rather than the yolk sac.

\section{Hemogenic endothelium and hematopoietic stem cell emergence}

The first blood progenitors endowed with LTR potential emerge in the AGM region of the E10.5 mouse embryo [17, 22, 51]. Further investigation revealed that the DA and other major arteries of the embryo were the source of the first HSCs [52, 53].

Hematopoietic cells have for a long time been associated with the embryonic DA. Clusters of hematopoietic cells attached to the ventral wall of the DA were first described over a century 
ago and they have been found in all vertebrates studied to date. This indicates that their formation is a very well conserved process. Further research showed that human and chick intra-aortic clusters contain multipotent progenitor cells $[20,54,55]$ suggesting that the first HSCs are contained within these intra-aortic clusters. Indeed, cells isolated from mouse intraaortic clusters have LTR potential [53]. Intriguingly, blood clusters are found associated with both the dorsal and ventral walls of the mouse DA. However, transplantations carried out with dissected dorsal or ventral halves of the DA confirmed that only ventrally associated clusters contain HSCs $[21,56]$.

The intimate association of the intra-aortic clusters with the endothelial lining of the DA raised the possibility that HSCs derive from endothelial cells. The endothelial origin of the blood intra-aortic clusters was first suggested at the beginning of the $20^{\text {th }}$ century and the term 'hemogenic endothelium' was coined to describe this progenitor cell [57-59]. Nevertheless, only recent lineage labelling and in vivo imaging experiments have provided direct evidence for the endothelial origin of HSCs. Labelling chick DA cells either via LDL uptake or viral transfection strongly suggested that intra-aortic clusters derive from endothelial cells $[60,61]$. Additionally, human endothelial cells isolated from the embryonic DA are capable of differentiating into myeloid and lymphoid progeny when cultured in vitro [62]. Furthermore, labeling endothelial cells via conditional transgenesis techniques, demonstrated that mouse intra-aortic clusters and all hematopoietic cells in the adult bone marrow derive from endothelial precursors [63-65]. Concomitantly, it was shown that mesenchymal cells surrounding the DA do not give rise to blood cells [64], an important issue since the transgenic lines used to isolate cells from intra-aortic clusters also labeled cells within the mesenchyme [52, 53]. Most importantly, live imaging in zebrafish embryos and in vitro 
cultured mouse AGM explants demonstrated that endothelial cells localised in the ventral wall of the DA undergo morphological and phenotypical changes as they acquire hematopoietic potential to eventually generate budding hematopoietic cells, a process termed the endothelial to hematopoietic transition (EHT)[30, 66-70].

At the genetic level, a number of transcription factors (TFs) are required for HSC development, including members of the ETS and Gata family of TFs[71]. However, due to their multiple functions in hematopoiesis and other developmental processes, it has been difficult to establish whether they play a direct role in EHT giving rise to HSCs. Nevertheless, three TFs have been shown to be directly involved in EHT, Gata2, Scl and Runx1. Gata2 and Scl are recurrently used during blood development and play multiple context dependent roles and they are required for both embryonic and adult blood lineages [72-78] and their interaction with ETS TFs generate self-regulated genetic circuits controlling multiple aspects of hematopoiesis [79-82]. Gata2 is regarded as a regulator of $s c l$ expression while $\mathrm{Scl}$ is a key determinant of the hematopoietic fate by controlling hemangioblasts specification $[72,74,81,83]$. In the mouse, Gata2 and Scl null embryos die before the emergence of HSCs, hampering the analysis of their function in EHT [77, 78]. Gata2 is one of the most studied blood TFs and multiple enhancers controlling its expression in different cells types, including non-hematopoietic and endothelial cells, have been identified. Importantly, a Gata2 cis-element (+9.5) critical for its expression in the mouse AGM has recently been isolated and used to generate tamoxifen-inducible Cre mice and delete Gata2 specifically in endothelial cells, which results in the loss of HSCs in the fetal liver [84]. Deletion of this enhancer in its own, reduces the expression of a number of key blood TFs, including $s c l$ and runxl, in the AGM and abrogates the capacity of hemogenic endothelium to 
generate HSCs [85]. Moreover, specific deletion of Gata2 in VE-Cadherin-expressing endothelial cells before the generation of HSCs abrogates the generation of intra-aortic clusters and LTR HSCs [86]. In this Gata2 mutant, the DA endothelium forms normally and no increase in apoptosis is observed; importantly, cKit expression analysis showed the persistence of cells with an endothelial phenotype expressing cKit, suggesting that hemogenic endothelium formed but is unable to generate intra-aortic clusters [86], thus indicating a direct role for Gata2 in EHT. The pivotal role of Scl in definitive blood specification is reflected by the lack of hemangioblast specification and the physical absence of the DA in Xenopus and zebrafish embryos depleted of this TF [83, 87]. Interestingly, two Scl isoforms exist in the zebrafish, Scl $\alpha$ and $\operatorname{Scl} \beta$, and they exhibit differential expression within the DA, Scl $\beta$ is expressed before EHT while Scl $\alpha$ is expressed later in emerging HSCs. Functional analysis indicates that, while Scl $\alpha$ is involved in the maintenance of newly formed HSCs, Scl $\beta$ controls the generation of HSCs upstream of Runx1, in the absence of Scl $\beta$ hemogenic endothelium dies before undergoing EHT [88].

In contrast to Gata2 and Scl, Runx1has minimal roles in embryonic blood but is absolutely required for definitive blood and definitive HSC emergence: no HSCs are generated in mouse AGM when Runx1 is deleted [44, 89-93]. Furthermore, specific deletion of Runx1 in VECadherin- and Vav1-expressing cells, demonstrated that Runx1's requirement is specific to HSC generation and not for their maintenance [94-96]. In addition, Runx1 is required for the emergence of multipotent blood progenitors in the yolk sac, suggesting that all blood lineages deriving from hemogenic endothelium, including the Ly6a-expressing hemogenic endothelium which gives rise to HSCs, require Runx1 and, therefore, it was postulated that Runx1 regulates EHT $[90,94,97]$. Indeed, this was confirmed when EHT was analysed by 
live imaging in kdrl-GFP+ zebrafish: while in uninjected embryos hemogenic endothelium undergo EHT to form nascent HSCs, in Runx1-depleted embryos EHT was aborted and the prospective nascent HSCs die[66]. Thus, Runx1 appears to be the most important blood TF controlling EHT but how it achieves this is not known [94]. Similarly, hemogenic endothelium in ES cells has been characterised, and imaged, and its formation is also dependent on Runx1 $[98,99]$. In summary, it is currently accepted that HSCs differentiate from hemogenic endothelium through EHT. Nevertheless, the molecular mechanism of EHT is yet to be elucidated.

\section{Origins and development of the dorsal aorta}

HSCs emerge from the endothelium of the DA. Thus, an understanding of the origins and development of the DA is necessary for the elucidation of the mechanism involved in the programing of HSCs. The DA is the main axial artery of the embryo and in human, mouse and chick embryos it forms from the fusion of the preceding paired DA. Very little is known about the formation and maturation of the DA. It is known, however, that a midline/notochord BMP signalling regime dictates the timing of their fusion [100] but the signalling regulating their migration to the midline is not understood. It is also known that Notch signalling plays several key roles in the development/maturation of the DA [101, 102]. Notch1, Notch4 and Dll4 are located at the top of the signaling cascade regulating arterial specification [101-106], repression of the venous fate [107-109] and vessel lumenisation [110]. Importantly, Notch appears to initiate the specification of hemogenic endothelium in the DA by controlling runx1 expression [111-115]. The role of Notch is evolutionarily conserved since it also controls the expression of the Runx1 homolog, lozenge, in 
Droshophila lymph gland hematopoiesis [116]. In the mouse DA, HSC programing by Notch involves Gata2, Jagged1, Hes1 and Hes5 [117-119]. Interestingly, in the zebrafish, Wnt16 signaling has been shown to indirectly regulate HSC emergence through the Notch ligands, delta like $\mathrm{C}$ (DllC) and delta like D (DllD): expression of $d l l C$ and $d l l D$ in the somites is required for HSC emergence and this expression is regulated by Wnt16 [120, 121]. Lastly, only the ventral wall of the DA generates HSC; how this polarisation is established is poorly understood. However, at least in the zebrafish, reciprocal BMP-hedgehog gradients appear to be critical [122] (Figure 3).

Regarding the localisation of the DA progenitors, it has been described in the chick that the dorsal wall derives from the somites while the ventral wall derives from splanchnic mesoderm [123]. Thus, it was proposed that the dual ontogeny of the DA explained the polarisation of $\mathrm{HSC}$ emergence, particularly because intra-aortic cluster formation terminates as the splanchnic endothelium is replaced by somite derived endothelium [124, 125]. However, there is no evidence in the mouse, Xenopus or zebrafish indicating that the somites contribute to the DA of the trunk, where HSCs emerge. Indeed, gene expression analysis by RNA in situ hybridisation and lineage labeling experiments in the mouse have indicated that all the endothelium of the DA, including the hemogenic endothelium, derives from lateral plate mesoderm $[63,65,126,127]$. Moreover, all the endothelium of the descending DA of adult mice derives from lateral plate mesoderm [63]. Similarly, in Xenopus and zebrafish, the lateral plate mesoderm gives rise to endothelial precursors which migrate to the midline to form the DA [50,112, 128-130](Figure 3). Thus, polarisation of the DA cannot be explained by differential ontogeny of the dorsal and ventral walls. 
Although the mouse DA originates from lateral plate mesoderm, the exact location and the nature of its progenitors are unknown. In contrast, lateral plate mesoderm populations of hemangioblasts giving rise to the DA have been described in the zebrafish and Xenopus [71, $74,83,131,132]$. In the zebrafish these hemangioblasts are intermingled with hemangioblasts giving rise to embryonic blood progenitors, making their study more difficult. Fortunately, in Xenopus, DLP hemangioblasts are physically and temporally separated from their embryonic blood counterparts. Therefore, the gene regulatory network (GRN) controlling their specification can be established [83, 133]. The current GRN shows that ETS TFs and Gata2 are key players in DLP hemangioblast specification and, critically, that synergism between VEGFA-dependent and VEGFA-independent pathways is required for tall/scl activation in the DLP [83, 134]. This GRN also shows the critical role of VEGFA produced in the somites [135] and a double-negative gate controlled by miR-142-3p [133] in the initiation of the hemangioblast programme. Studies in Xenopus have also demonstrated, for the first time, that different VEGFA isoforms perform distinct and specific roles during HSC ontogeny [114] and further support the view that the somites act as a signalling centre in HSC programing [101, 112, 120, 134].

\section{The hemangioblast}

The development of hematopoietic cells is intimately associated with that of endothelial cells. In the late $19^{\text {th }}$ century, embryologists observed that the chick yolk sac blood islands differentiate from clusters of cells surrounded by mesenchymal cells. Although cells within these clusters are initially indistinguishable from each other, as they differentiate into blood islands, the inner cells become erythrocytes while the outer cells become endothelial cells 
(Figure 3a). This morphological observation suggested that blood and endothelial cells may derive from a bi-potential common progenitor cell, the hemangioblast [136, 137]. More recently, genetic evidence has provided strong support for the existence of this bi-potential progenitor cell. In all models studied to date, including Drosophila [138], the presence of cells co-expressing numerous blood and endothelial genes has been described. These genes include key TFs such as gata2, etv2, fli1, Imo2 and tall/scl. Also, these cell populations have been shown to serve as blood and endothelial progenitors [71]. Furthermore, mutations such as zebrafish cloche [139] and the deletion of a number of genes (including flk1, etv2 and tall/scl) results in blood and endothelial developmental deficiencies [87, 140-143]. More importantly, clonal analysis in Drosophila and lineage labelling in the zebrafish and chick embryos have demonstrated that cells with the capacity to give rise to blood and endothelial progeny do exist in the embryo [138, 144-146].

To date, the strongest evidence for the hemangioblast comes from cultured embryonic stem (ES) cells. Under certain conditions, single Flk1+ ES cells can give rise to blood and endothelial progeny $[147,148]$. Similarly, Flk1+ cells isolated from the posterior primitive streak can give rise to equivalent colonies, indicating the existence of hemangioblasts in vivo [149]. However, the capacity of these cells to also give rise to smooth muscle cells has raised the possibility that these blast colony forming cells represent earlier cardiovascular progenitor cells rather than hemangioblasts.

In contradiction to the genetic and in vitro evidence for the hemangioblast, some in vivo lineage labeling experiments in a number of species appear to disagree with the existence of a bi-potential progenitor cell giving rise to both blood and endothelial cells. The formation of 
discrete blood islands in the chick yolk sac suggested a clonal origin of these structures.

However, in the mouse, rather than discrete blood islands, a band of differentiating blood and endothelial cells is observed [150] and therefore their origin is less likely to be of a clonal nature, as has indeed been suggested by lineage labelling experiments [151]. Nonetheless, the polyclonal nature of the mouse yolk sac blood islands does not negate the existence of hemangioblasts since some clones still gave rise to both blood and endothelial cells [151]. Similarly, cell tracking and explant studies in Xenopus appear to indicate that the VBI contains unilineage rather than bi-potential progenitor cells [152]. However co-expression data suggest that these experiments may have been performed after the blood and endothelial lineages had already segregated [153].

The rarity of hemangioblasts in vivo might be due to the technical challenges required to capture these transient progenitors but could also be a reflection of the developmental constraints imposed by their environment (Figure 2A). For example, it is unclear whether cells within the yolk sac blood islands, or their progenitors, undergo cell division. This information is critical since a non-dividing hemangioblast cannot give rise to two daughter cells with different fates but rather can only differentiate into one or the other lineage. Signaling may greatly influence not only hemangioblast fate decisions but also cell division, as indicated when Flt1 was deleted. Flt1 binds to and limits the amount of VEGFA signaling through Flk1 but in its absence Flk1 is hyperactivated and this results in excessive proliferation of hemangioblasts [154]. Also, over-expression of the critical hemangioblast gene, tall/scl, appears to bypass some of these developmental constraints since it induces an overproduction of hemangioblasts which are incapable of differentiating into mature blood cells unless other factors are present [155]. Thus, when these cells are isolated and cultured, 
their developmental constraints are disrupted, they undergo cell division and express their bipotentiality (Figure 3B). Ultimately, in vivo single cell labelling and live imaging experiments, similar to those performed for the DA hemogenic endothelium, are required to settle the existence of the hemangioblast. In addition, these cells may need to be transplanted into an environment that permits the expression of their bi-potential nature.

\section{Lineage relationship between blood and cardiac cells}

Inhibition of Wnt signaling in Xenopus embryos induces cardiac expression in ventral mesoderm at the expense of blood cells [156]. Because Wnt signaling plays important roles in mesoderm patterning it was believed that the altered cell fate was due to mesodermal patterning defects rather than reflecting a lineage relationship. Enforced expression of blood TFs in the zebrafish results in the conversion of cardiac progenitors into blood [155] and, together with later loss of function data [157], it was proposed that the cardiac programme can be antagonised by blood TFs. More recent experiments in zebrafish have shown that there is in fact cross-antagonism between these two programmes [158]. Meanwhile, studies in cultured ES cells demonstrated that Flk1+ cells can give rise to cardiomyocytes, suggesting that blood and cardiac cells may share a common precursor in this system [159].

In vivo studies in Xenopus and zebrafish added further evidence for this common progenitor cell and established that cardiac Gata factors are also essential for the hemangioblast lineage, and that the cross-antagonism between the hemangioblast and cardiac TFs is resolved in favour of cardiac differentiation by FGF $[157,158,160]$. More recently, in the ES system, an etv2-scl axis has emerged as key for the repression of cardiac genes and fate. In Flk1+ 
mesodermal progenitors, etv2 positively regulates hematopoietic and endothelial genes while repressing cardiac genes and Wnt signaling [161]. Conversely, in the absence of etv2, endothelial and endocardial cells differentiate into cardiomyocytes [162]. Similarly, in the absence of scl, hemogenic endothelium and endocardial cells activate the cardiac programme [163]. In Scl-deficient mouse embryos, the endocardium is disorganised due to precocious differentiation into cardiomyocytes which leads to ectopic cardiogenesis; Scl repression of cardiogenesis is cell-intrinsic and does so by repressing Wnt antagonist which promote cardiogenesis [163]. This suggests that active repression of the cardiac programme is required even in advanced stages of endothelial and endocardial differentiation.

\section{The Physiology of HSC emergence}

In recent years, common physiological requirements between adult bone marrow HSCs and emerging HSCs in the DA have been found. Hoechst33342 perfusion from blood vessels, which presumably correlates with oxygen levels, suggested that HSCs reside in the most hypoxic regions of the bone marrow and this was confirmed by pimonidazole staining, an hypoxia indicator, which demonstrated that the most potent HSCs are located in niches with low oxygen tension $[164,165]$. Furthermore, it has been shown that HSCs maintain intrinsic hypoxia by stabilizing HIF1 $\alpha$, which maintains cell cycle quiescence and its deletion decreases quiescent HSC numbers [166]. In addition, VEGFA, a factor required in a cellintrinsic manner for HSC maintenance [167], contains hypoxia binding elements in its regulatory regions that, when mutated, decrease VEGFA expression levels, thereby impairing HSC function [168]. Pimonidazole staining in E10 mouse embryos showed that the endothelium of the DA and intra-aortic clusters is also hypoxic, and deletion of HIF1 $\alpha$ in VE- 
Cadherin-expressing cells reduced the number of intra-aortic clusters. However, interestingly, some HSCs were still generated, indicating that not all HSCs are dependent on this TF[169].

Exposure of zebrafish embryos to $1 \%$ D-glucose increases the number of transplantable HSCs generated in the DA without systemic alteration in growth and vascular development: cell proliferation increased only in the AGM, indicating that the number of HSCs generated in the embryo depend on hemogenic endothelium nutrition and metabolic status [170]. This enhancement of HSC production is not due to alterations in cardiovascular function as glucose retained the ability to increase HSC number in the silent heart mutant, which lacks blood flow due to a cardiac defect. It is however dependent on Runx1 since the number of $c M y b$ expressing cells do not increase when Runx1 morphants are treated with glucose[170]. Further analysis indicates that stem cell numbers depend on glucose intake because numbers of runxl and $c M y b$ expressing cells decrease when the glucose transporter, glut1, is downregulated. Increased glucose intake increases glycolysis that produces reactive oxygen species which in turn stabilize HIF1 $\alpha$. The role of HIF1 $\alpha$ as the mediator of the glucose response was confirmed by the loss of runxl expression in the DA of HIF1 $\alpha$ morphants, this loss is concomitant to down-regulation of the HIF1 $\alpha$ target gene, vefgA, and the HIF1 $\alpha$ phenotype can be rescued by exogenous VEGFA[170]. HIF1 $\alpha$ is considered as master regulator of metabolism because it regulates both glycolysis and mitochondrial respiration, and in bone marrow HSCs, which intrinsically maintain HIF1 $\alpha$ expression [166], it induces glycolysis while repressing mitocholdrial respiration in such a way that the most potent LTR HSCs are enriched in the low mitochondria / glycolysis-dependent population [171]. Thus, it is clear that in both the bone marrow and AGM niches, in addition to sensing oxygenation levels, 
HIF1 $\alpha$ mediates the metabolic response to glucose and, in the AGM, controls the number of emerging HSCs.

Blood flow is an important physiological process that provides biomechanical forces which influence developing blood vessels. For example, it influences blood vessel diameter and determines their arterial-venous fate. Manipulation of the flow pattern can transform arteries into veins and veins into arteries [172], indicating that flow has the capacity to override the genetic programming of these vessels. A role for biomechanical forces in hematopoiesis has recently been reported in in vitro and in vivo systems. In differentiating ES cells shear stress increases the expression of runxl in CD41+cKit+ progenitors and concomitantly enhances their colony-forming potential [173]. Shear stress also increases hematopoietic expression in the mouse AGM as well as its colony-forming potential, and this is dependent on nitric oxide (NO), a mediator of shear stress [173]. A chemical screen in the zebrafish also showed that compounds modulating blood flow regulate HSC formation [174]. The role of blood flow in HSC formation was further confirmed by the loss of HSCs in the silent heart mutant, which lacks a heartbeat due to a mutation in the heart contractile machinery, and, importantly, NO rescues the loss of HSCs in this mutant [174]. Furthermore, it was determined that zebrafish NO synthase 1, nos 1 , is required cell autonomously for HSC formation and that NO signalling acts downstream of Wnt signaling [174]. Interestingly, in the mouse, HSC formation is not affected in Nos1-deficient embryos instead their numbers were reduced in Nos3 mutants [174], this, nevertheless, confirmed the conservation of NO signaling in HSC generation. More recently, in the zebrafish, it has been demonstrated that blood flow, rather than initiating runxl expression in the DA, is required for its maintenance [175]. It has also been determined that Klf2a directly binds the promoters of nos genes, that it is capable of 
recuing HSC generation in mutants lacking heartbeat and that its down-regulation phenocopies these mutants [175]. The relationship between this Klf2a genetic cascade and Wnt signaling is yet to be determined.

Prostaglandin signaling is essential for tissue homeostasis and it is implicated in cancers and other pathologies [176]. In the hematopoietic system, prostaglandin E1 and E2 stimulates HSC proliferation both in vitro and in the bone marrow, as measured by spleen colonyforming units [177, 178]. Ex-vivo treatment of bone marrow or cord blood HSCs with prostaglandin E2 (PGE2), or its long acting analog, dmPGE2, stimulates their proliferation and differentiation as well as enhancing their transplantation efficiency [179-182]. Recent studies in the zebrafish have revealed important roles for prostaglandin signaling in developmental hematopoiesis. Stimulation of the prostaglandin pathway with PGE2 increases the proliferation of progenitor cells in the thymus whereas its repression has opposite effects [183]. Furthermore, the absence of a single prostaglandin receptor, EP4, results in the loss of lymphoid progenitors in the thymus [183]. Prostaglandin signaling has a similar function in nascent HSCs in the zebrafish DA, chemical screening showed that compounds which enhance prostaglandin synthesis increase HSC numbers whereas those blocking it reduce them [184]. Also, PGE2 improved kidney marrow recovery after irradiation injury in adult fish [184]. Mechanistically, PGE2 stabilises $\beta$-catenin to activate Wnt signaling and inhibition of prostaglandin signaling leads to Wnt signaling and HSC emergence deficiencies [185]. More recently, it has been shown that dmPGE2 enhances the migration and homing capacity of bone marrow HSCs by inducing the activity of HIF1 $\alpha$ [186], whether Wnt signaling is involved in this process is unknown. The involvement of Wnt signaling in the regulation of HIF1 $\alpha$ in the AGM is also unknown. Furthermore, retinoic acid receptor (RAR) 
signaling, which positively regulates HSC maintenance in vitro [187], has been shown to regulate hemogenic endothelium and HSC emergence in the mouse AGM and does so by interacting with $\beta$-catenin to repress Wnt signaling [188]. Thus, Wnt signaling appears to be both required and detrimental to HSC generation in the AGM. Further studies are required to clarify its role(s) as well as the genetic interaction between blood flow, HIF1 $\alpha$, prostaglandin, retinoic acid and metabolism.

The inflammatory and immune signaling pathways could also be involved in HSC emergence/maintenance. Gata2 has recently been associated with the inflammatory GRN [189]. Gata2 ChIP-seq analysis in human endothelial cells showed that inflammatory genes are significant Gata2 targets and that Gata2 consistently occupied sites with motifs bound by activator protein-1 (AP-1), a major regulator of inflammatory genes, and its binding partners, c-JUN and c-FOS. Gata2 is not required for AP-1 chromatin occupancy, nevertheless, it stimulates AP-1-dependent transactivation [189]. Although this analysis was carried out in HUVECs, it could indicate that components of the inflammatory GRN might be involved in the development of embryonic endothelial and hematopoietic cells, and potentially, in HSC emergence. In a recent study, Stat 1 has emerged as a potential regulator of definitive hematopoiesis [190]. By comparing primitive and definitive erythrocyte lineage-specific data sets, a striking Stat1-related expression signature was found differentially expressed in the definitive lineage, including the interferon signaling pathway[190]. Since this blood lineage derives from hemogenic endothelium, Stat1 and interferon signaling might be implicated in EHT and HSC emergence but this is yet to be determined. 
The AGM niche produces growth factors that are essential for HSC emergence and attempts to isolate stromal cells capable of sustaining HSC emergence have been made [191-196]. However, still little is known about the role of the AGM niche and the role of non-cell autonomous factors in the generation of HSCs. The BMP, hedgehog, Wnt and Notch signaling pathways are active in the mouse DA mesenchyme and have been shown to influence HSC emergence through unknown transcriptional networks [115, 197-199]. Nonetheless, Gata3 has recently emerged as a key non-cell autonomous TF required for HSC emergence [200]. Gata3 was initially reported to be required for fetal liver hematopoiesis and the development of the neural system [201]. Further, analysis of Gata3 deficient embryos demonstrated its requirement for HSC emergence [200]. Interestingly, while rare DA endothelial cells expressed Gata3, numerous cells within the mesenchyme expressed it and further analysis showed that Gata3's role in HSC emergence is indeed non-cell autonomous. Furthermore, Gata3 is strongly expressed and required for sympathetic neuron development, and these neurons produce catecholamines which serve as ligands for adrenergic receptors expressed by hemogenic endothelium, thus, stimulating the generation of HSCs [200]. Undoubtedly, the AGM creates a unique microenvironment for the generation of HSCs and its study is required for an adequate understanding of HSC ontogeny.

\section{Perspectives}

Model organisms have contributed enormously to our understanding of developmental hematopoiesis and undoubtedly will continue to do so for the elucidation of the molecular mechanism of HSC ontogeny, and to resolve current controversies. An advantageous treat of the zebrafish model is its amenability to large scale chemical and genetic screenings. In 
addition, the optical clarity of the zebrafish embryo makes it ideal for live imaging which would facilitate the analysis of the migratory pathways and other cellular processes undertaken by emerging HSCs and their progeny. In its turn, the Xenopus model is wellsuited for the study of the mechanisms involved in the programing of HSCs, from hemangioblasts in the lateral plate to the generation of hemogenic endothelium in the DA. The advent of new technologies such as TALEN and CRISPR genome editing, new imaging strategies and high throughput genome wide analysis, including epigenetic studies, will greatly enhance the potential of these model organisms.

\section{Acknowledgements}

This Work was supported by the UK MRC, the British Heart Foundation (RM) and the Oxford BHF Centre of Research Excellence (RM, RP).

\section{References}

[1] Medvinsky A, Rybtsov S, Taoudi S. Embryonic origin of the adult hematopoietic system: advances and questions. Development. 2011;138:1017-1031.

[2] Johnson GR, Moore MA. Role of stem cell migration in initiation of mouse foetal liver haemopoiesis. Nature. 1975;258:726-728.

[3] Moore MA, Metcalf D. Ontogeny of the haemopoietic system: yolk sac origin of in vivo and in vitro colony forming cells in the developing mouse embryo. British journal of haematology. 1970;18:279-296.

[4] Dzierzak E, Medvinsky A, de Bruijn M. Qualitative and quantitative aspects of haematopoietic cell development in the mammalian embryo. Immunology today. 1998;19:228-236.

[5] Fontaine-Perus JC, Calman FM, Kaplan C, Le Douarin NM. Seeding of the 10-day mouse embryo thymic rudiment by lymphocyte precursors in vitro. J Immunol. 1981;126:2310-2316.

[6] Owen JJ, Ritter MA. Tissue interaction in the development of thymus lymphocytes. The Journal of experimental medicine. 1969;129:431-442.

[7] LeDouarin NM, Jotereau FV. Origin and renewal of lymphocytes in avian embryo thymuses studied in interspecific combinations. Nature: New biology. 1973;246:25-27. 
[8] Pardanaud L, Yassine F, Dieterlen-Lievre F. Relationship between vasculogenesis, angiogenesis and haemopoiesis during avian ontogeny. Development. 1989;105:473-485.

[9] Houssaint E. Differentiation of the mouse hepatic primordium. II. Extrinsic origin of the haemopoietic cell line. Cell differentiation. 1981;10:243-252.

[10] Dieterlen-Lievre F. On the origin of haemopoietic stem cells in the avian embryo: an experimental approach. Journal of embryology and experimental morphology. 1975;33:607619.

[11] Dieterlen-Lievre F, Martin C. Diffuse intraembryonic hemopoiesis in normal and chimeric avian development. Developmental biology. 1981;88:180-191.

[12] Kau CL, Turpen JB. Dual contribution of embryonic ventral blood island and dorsal lateral plate mesoderm during ontogeny of hemopoietic cells in Xenopus laevis. J Immunol. 1983;131:2262-2266.

[13] Maeno M, Todate A, Katagiri C. The Localization of Precursor Cells for Larval and Adult Hematopoietic-Cells of Xenopus-Laevis in 2 Regions of Embryos. Development Growth \& Differentiation. 1985;27:137-148.

[14] Bechtold TE, Smith PB, Turpen JB. Differential stem cell contributions to thymocyte succession during development of Xenopus laevis. J Immunol. 1992;148:2975-2982.

[15] Chen XD, Turpen JB. Intraembryonic origin of hepatic hematopoiesis in Xenopus laevis. J Immunol. 1995;154:2557-2567.

[16] Smith PB, Flajnik MF, Turpen JB. Experimental analysis of ventral blood island hematopoiesis in Xenopus embryonic chimeras. Developmental biology. 1989;131:302-312.

[17] Medvinsky AL, Samoylina NL, Muller AM, Dzierzak EA. An early pre-liver intraembryonic source of CFU-S in the developing mouse. Nature. 1993;364:64-67.

[18] Tavian M, Robin C, Coulombel L, Peault B. The human embryo, but not its yolk sac, generates lympho-myeloid stem cells: mapping multipotent hematopoietic cell fate in intraembryonic mesoderm. Immunity. 2001;15:487-495.

[19] Cumano A, Dieterlen-Lievre F, Godin I. Lymphoid potential, probed before circulation in mouse, is restricted to caudal intraembryonic splanchnopleura. Cell. 1996;86:907-916.

[20] Tavian M, Coulombel L, Luton D, Clemente HS, Dieterlen-Lievre F, Peault B. Aortaassociated CD34+ hematopoietic cells in the early human embryo. Blood. 1996;87:67-72.

[21] Ivanovs A, Rybtsov S, Anderson RA, Turner ML, Medvinsky A. Identification of the niche and phenotype of the first human hematopoietic stem cells. Stem cell reports.

2014;2:449-456.

[22] Medvinsky A, Dzierzak E. Definitive hematopoiesis is autonomously initiated by the AGM region. Cell. 1996;86:897-906.

[23] Boiers $C$, Carrelha J, Lutteropp M, et al. Lymphomyeloid contribution of an immunerestricted progenitor emerging prior to definitive hematopoietic stem cells. Cell stem cell. 2013;13:535-548.

[24] Yoshimoto M, Montecino-Rodriguez E, Ferkowicz MJ, et al. Embryonic day 9 yolk sac and intra-embryonic hemogenic endothelium independently generate a B-1 and marginal zone progenitor lacking B-2 potential. Proceedings of the National Academy of Sciences of the United States of America. 2011;108:1468-1473. 
[25] Yoder MC, Hiatt K, Dutt P, Mukherjee P, Bodine DM, Orlic D. Characterization of definitive lymphohematopoietic stem cells in the day 9 murine yolk sac. Immunity. 1997;7:335-344.

[26] Murayama E, Kissa K, Zapata A, et al. Tracing hematopoietic precursor migration to successive hematopoietic organs during zebrafish development. Immunity. 2006;25:963975.

[27] Kissa K, Murayama E, Zapata A, et al. Live imaging of emerging hematopoietic stem cells and early thymus colonization. Blood. 2008;111:1147-1156.

[28] Jin H, Sood R, Xu J, et al. Definitive hematopoietic stem/progenitor cells manifest distinct differentiation output in the zebrafish VDA and PBI. Development. 2009;136:647654.

[29] Monteiro R, Pouget C, Patient R. The gata1/pu.1 lineage fate paradigm varies between blood populations and is modulated by tif1gamma. The EMBO journal. 2011;30:1093-1103.

[30] Bertrand JY, Chi NC, Santoso B, Teng S, Stainier DY, Traver D. Haematopoietic stem cells derive directly from aortic endothelium during development. Nature. 2010;464:108111.

[31] Traver D, Paw BH, Poss KD, Penberthy WT, Lin S, Zon LI. Transplantation and in vivo imaging of multilineage engraftment in zebrafish bloodless mutants. Nature immunology. 2003;4:1238-1246.

[32] Traver D, Winzeler A, Stern HM, et al. Effects of lethal irradiation in zebrafish and rescue by hematopoietic cell transplantation. Blood. 2004;104:1298-1305.

[33] Ma D, Zhang J, Lin HF, Italiano J, Handin RI. The identification and characterization of zebrafish hematopoietic stem cells. Blood. 2011;118:289-297.

[34] Turpen JB, Smith PB. Location of hemopoietic stem cells influences frequency of lymphoid engraftment in Xenopus embryos. J Immunol. 1989;143:3455-3460.

[35] Rollins-Smith LA, Blair P. Contribution of ventral blood island mesoderm to hematopoiesis in postmetamorphic and metamorphosis-inhibited Xenopus laevis. Developmental biology. 1990;142:178-183.

[36] Palis J, Malik J, McGrath KE, Kingsley PD. Primitive erythropoiesis in the mammalian embryo. The International journal of developmental biology. 2010;54:1011-1018.

[37] Palis J, Robertson S, Kennedy M, Wall C, Keller G. Development of erythroid and myeloid progenitors in the yolk sac and embryo proper of the mouse. Development. 1999;126:5073-5084.

[38] Rampon C, Huber P. Multilineage hematopoietic progenitor activity generated autonomously in the mouse yolk sac: analysis using angiogenesis-defective embryos. The International journal of developmental biology. 2003;47:273-280.

[39] Lux CT, Yoshimoto M, McGrath K, Conway SJ, Palis J, Yoder MC. All primitive and definitive hematopoietic progenitor cells emerging before E10 in the mouse embryo are products of the yolk sac. Blood. 2008;111:3435-3438.

[40] Bertrand JY, Kim AD, Violette EP, Stachura DL, Cisson JL, Traver D. Definitive hematopoiesis initiates through a committed erythromyeloid progenitor in the zebrafish embryo. Development. 2007;134:4147-4156. 
[41] Sugiyama D, Ogawa $M$, Nakao $K$, et al. B cell potential can be obtained from precirculatory yolk sac, but with low frequency. Developmental biology. 2007;301:53-61.

[42] Li Z, Lan Y, He W, et al. Mouse embryonic head as a site for hematopoietic stem cell development. Cell stem cell. 2012;11:663-675.

[43] Nakano H, Liu X, Arshi A, et al. Haemogenic endocardium contributes to transient definitive haematopoiesis. Nature communications. 2013;4:1564.

[44] Dzierzak E, Speck NA. Of lineage and legacy: the development of mammalian hematopoietic stem cells. Nature immunology. 2008;9:129-136.

[45] Yoder MC, Hiatt K, Mukherjee P. In vivo repopulating hematopoietic stem cells are present in the murine yolk sac at day 9.0 postcoitus. Proceedings of the National Academy of Sciences of the United States of America. 1997;94:6776-6780.

[46] Toles JF, Chui DH, Belbeck LW, Starr E, Barker JE. Hemopoietic stem cells in murine embryonic yolk sac and peripheral blood. Proceedings of the National Academy of Sciences of the United States of America. 1989;86:7456-7459.

[47] Turpen JB, Kelley CM, Mead PE, Zon LI. Bipotential primitive-definitive hematopoietic progenitors in the vertebrate embryo. Immunity. 1997;7:325-334.

[48] Gordon-Keylock S, Sobiesiak M, Rybtsov S, Moore K, Medvinsky A. Mouse extraembryonic arterial vessels harbor precursors capable of maturing into definitive HSCs. Blood. 2013;122:2338-2345.

[49] Samokhvalov IM, Samokhvalova NI, Nishikawa S. Cell tracing shows the contribution of the yolk sac to adult haematopoiesis. Nature. 2007;446:1056-1061.

[50] Ciau-Uitz A, Walmsley M, Patient R. Distinct origins of adult and embryonic blood in Xenopus. Cell. 2000;102:787-796.

[51] Muller AM, Medvinsky A, Strouboulis J, Grosveld F, Dzierzak E. Development of hematopoietic stem cell activity in the mouse embryo. Immunity. 1994;1:291-301.

[52] de Bruijn MF, Speck NA, Peeters MC, Dzierzak E. Definitive hematopoietic stem cells first develop within the major arterial regions of the mouse embryo. The EMBO journal. 2000;19:2465-2474.

[53] de Bruijn MF, Ma X, Robin C, Ottersbach K, Sanchez MJ, Dzierzak E. Hematopoietic stem cells localize to the endothelial cell layer in the midgestation mouse aorta. Immunity. 2002;16:673-683.

[54] Cormier F, Dieterlen-Lievre F. The wall of the chick embryo aorta harbours M-CFC, G-CFC, GM-CFC and BFU-E. Development. 1988;102:279-285.

[55] Cormier F, de Paz P, Dieterlen-Lievre F. In vitro detection of cells with monocytic potentiality in the wall of the chick embryo aorta. Developmental biology. 1986;118:167175.

[56] Taoudi S, Medvinsky A. Functional identification of the hematopoietic stem cell niche in the ventral domain of the embryonic dorsal aorta. Proceedings of the National Academy of Sciences of the United States of America. 2007;104:9399-9403.

[57] Jordan HE. Aortic Cell Clusters in Vertebrate Embryos. Proceedings of the National Academy of Sciences of the United States of America. 1917;3:149-156.

[58] Jordan HE. Evidence of hemogenic capacity of endothelium. Anat Rec. 1916;10:417420. 
[59] Emmel VE. The cell clusters in the dorsal aorta of mammalian embryos. Am J Anat. 1916;19:401-421.

[60] Jaffredo T, Gautier R, Brajeul V, Dieterlen-Lievre F. Tracing the progeny of the aortic hemangioblast in the avian embryo. Developmental biology. 2000;224:204-214.

[61] Jaffredo T, Gautier R, Eichmann A, Dieterlen-Lievre F. Intraaortic hemopoietic cells are derived from endothelial cells during ontogeny. Development. 1998;125:4575-4583.

[62] Oberlin E, Tavian M, Blazsek I, Peault B. Blood-forming potential of vascular endothelium in the human embryo. Development. 2002;129:4147-4157.

[63] Wasteson P, Johansson BR, Jukkola T, et al. Developmental origin of smooth muscle cells in the descending aorta in mice. Development. 2008;135:1823-1832.

[64] Zovein AC, Hofmann JJ, Lynch M, et al. Fate tracing reveals the endothelial origin of hematopoietic stem cells. Cell stem cell. 2008;3:625-636.

[65] Zovein AC, Turlo KA, Ponec RM, et al. Vascular remodeling of the vitelline artery initiates extravascular emergence of hematopoietic clusters. Blood. 2010;116:3435-3444.

[66] Kissa K, Herbomel P. Blood stem cells emerge from aortic endothelium by a novel type of cell transition. Nature. 2010;464:112-115.

[67] Boisset JC, van Cappellen W, Andrieu-Soler C, Galjart N, Dzierzak E, Robin C. In vivo imaging of haematopoietic cells emerging from the mouse aortic endothelium. Nature. 2010;464:116-120.

[68] Mizuochi C, Fraser ST, Biasch K, et al. Intra-aortic clusters undergo endothelial to hematopoietic phenotypic transition during early embryogenesis. PloS one. 2012;7:e35763.

[69] Swiers G, Baumann C, O'Rourke J, et al. Early dynamic fate changes in haemogenic endothelium characterized at the single-cell level. Nature communications. 2013;4:2924.

[70] Lam EY, Hall CJ, Crosier PS, Crosier KE, Flores MV. Live imaging of Runx1 expression in the dorsal aorta tracks the emergence of blood progenitors from endothelial cells. Blood. 2010;116:909-914.

[71] Ciau-Uitz A, Liu F, Patient R. Genetic control of hematopoietic development in Xenopus and zebrafish. International Journal of Developmental Biology. 2010;54:1139-1149.

[72] Porcher C, Liao EC, Fujiwara Y, Zon LI, Orkin SH. Specification of hematopoietic and vascular development by the bHLH transcription factor SCL without direct DNA binding. Development. 1999;126:4603-4615.

[73] Porcher C, Swat W, Rockwell K, Fujiwara Y, Alt FW, Orkin SH. The T cell leukemia oncoprotein SCL/tal-1 is essential for development of all hematopoietic lineages. Cell. 1996;86:47-57.

[74] Gering M, Rodaway AR, Gottgens B, Patient RK, Green AR. The SCL gene specifies haemangioblast development from early mesoderm. The EMBO journal. 1998;17:40294045.

[75] Liao EC, Paw BH, Oates AC, Pratt SJ, Postlethwait JH, Zon LI. SCL/Tal-1 transcription factor acts downstream of cloche to specify hematopoietic and vascular progenitors in zebrafish. Genes \& development. 1998;12:621-626.

[76] Dooley KA, Davidson AJ, Zon LI. Zebrafish scl functions independently in hematopoietic and endothelial development. Developmental biology. 2005;277:522-536. 
[77] Ng YK, George KM, Engel JD, Linzer DI. GATA factor activity is required for the trophoblast-specific transcriptional regulation of the mouse placental lactogen I gene. Development. 1994;120:3257-3266.

[78] Tsai FY, Keller G, Kuo FC, et al. An early haematopoietic defect in mice lacking the transcription factor GATA-2. Nature. 1994;371:221-226.

[79] Pimanda JE, Ottersbach K, Knezevic K, et al. Gata2, Fli1, and Scl form a recursively wired gene-regulatory circuit during early hematopoietic development. Proceedings of the National Academy of Sciences of the United States of America. 2007;104:17692-17697.

[80] Wilson NK, Foster SD, Wang X, et al. Combinatorial transcriptional control in blood stem/progenitor cells: genome-wide analysis of ten major transcriptional regulators. Cell stem cell. 2010;7:532-544.

[81] Gottgens B, Nastos A, Kinston S, et al. Establishing the transcriptional programme for blood: the $\mathrm{SCL}$ stem cell enhancer is regulated by a multiprotein complex containing Ets and GATA factors. The EMBO journal. 2002;21:3039-3050.

[82] Patterson LJ, Gering M, Eckfeldt CE, et al. The transcription factors Scl and Lmo2 act together during development of the hemangioblast in zebrafish. Blood. 2007;109:23892398.

[83] Ciau-Uitz A, Pinheiro P, Kirmizitas A, Zuo J, Patient R. VEGFA-dependent and independent pathways synergise to drive Scl expression and initiate programming of the blood stem cell lineage in Xenopus. Development. 2013;140:2632-2642.

[84] Lim KC, Hosoya T, Brandt W, et al. Conditional Gata2 inactivation results in HSC loss and lymphatic mispatterning. The Journal of clinical investigation. 2012;122:3705-3717.

[85] Gao X, Johnson KD, Chang Yl, et al. Gata2 cis-element is required for hematopoietic stem cell generation in the mammalian embryo. The Journal of experimental medicine. 2013;210:2833-2842.

[86] de Pater E, Kaimakis P, Vink CS, et al. Gata2 is required for HSC generation and survival. The Journal of experimental medicine. 2013;210:2843-2850.

[87] Patterson LJ, Gering M, Patient R. Scl is required for dorsal aorta as well as blood formation in zebrafish embryos. Blood. 2005;105:3502-3511.

[88] Zhen F, Lan Y, Yan B, Zhang W, Wen Z. Hemogenic endothelium specification and hematopoietic stem cell maintenance employ distinct Scl isoforms. Development.

2013;140:3977-3985.

[89] North TE, de Bruijn MF, Stacy T, et al. Runx1 expression marks long-term repopulating hematopoietic stem cells in the midgestation mouse embryo. Immunity. 2002;16:661-672.

[90] Tober J, Yzaguirre AD, Piwarzyk E, Speck NA. Distinct temporal requirements for Runx1 in hematopoietic progenitors and stem cells. Development. 2013;140:3765-3776.

[91] Okuda T, van Deursen J, Hiebert SW, Grosveld G, Downing JR. AML1, the target of multiple chromosomal translocations in human leukemia, is essential for normal fetal liver hematopoiesis. Cell. 1996;84:321-330.

[92] Wang Q, Stacy T, Miller JD, et al. The CBFbeta subunit is essential for CBFalpha2 (AML1) function in vivo. Cell. 1996;87:697-708.

[93] Wang Q, Stacy T, Binder M, Marin-Padilla M, Sharpe AH, Speck NA. Disruption of the $\mathrm{Cbfa} 2$ gene causes necrosis and hemorrhaging in the central nervous system and blocks 
definitive hematopoiesis. Proceedings of the National Academy of Sciences of the United States of America. 1996;93:3444-3449.

[94] Chen MJ, Yokomizo T, Zeigler BM, Dzierzak E, Speck NA. Runx1 is required for the endothelial to haematopoietic cell transition but not thereafter. Nature. 2009;457:887-891.

[95] North T, Gu TL, Stacy T, et al. Cbfa2 is required for the formation of intra-aortic hematopoietic clusters. Development. 1999;126:2563-2575.

[96] Cai X, Gaudet JJ, Mangan JK, et al. Runx1 loss minimally impacts long-term hematopoietic stem cells. PloS one. 2011;6:e28430.

[97] Chen MJ, Li Y, De Obaldia ME, et al. Erythroid/myeloid progenitors and hematopoietic stem cells originate from distinct populations of endothelial cells. Cell stem cell. 2011;9:541-552.

[98] Lancrin C, Sroczynska P, Stephenson C, Allen T, Kouskoff V, Lacaud G. The haemangioblast generates haematopoietic cells through a haemogenic endothelium stage. Nature. 2009;457:892-895.

[99] Eilken HM, Nishikawa S, Schroeder T. Continuous single-cell imaging of blood generation from haemogenic endothelium. Nature. 2009;457:896-900.

[100] Garriock RJ, Czeisler C, Ishii Y, Navetta AM, Mikawa T. An anteroposterior wave of vascular inhibitor downregulation signals aortae fusion along the embryonic midline axis. Development. 2010;137:3697-3706.

[101] Lawson ND, Vogel AM, Weinstein BM. sonic hedgehog and vascular endothelial growth factor act upstream of the Notch pathway during arterial endothelial differentiation. Developmental cell. 2002;3:127-136.

[102] Quillien A, Moore JC, Shin M, et al. Distinct Notch signaling outputs pattern the developing arterial system. Development. 2014.

[103] Lawson ND, Scheer N, Pham VN, et al. Notch signaling is required for arterial-venous differentiation during embryonic vascular development. Development. 2001;128:36753683.

[104] Krebs LT, Shutter JR, Tanigaki K, Honjo T, Stark KL, Gridley T. Haploinsufficient lethality and formation of arteriovenous malformations in Notch pathway mutants. Genes \& development. 2004;18:2469-2473.

[105] Benedito R, Trindade A, Hirashima M, et al. Loss of Notch signalling induced by DII4 causes arterial calibre reduction by increasing endothelial cell response to angiogenic stimuli. BMC developmental biology. 2008;8:117.

[106] Duarte A, Hirashima M, Benedito R, et al. Dosage-sensitive requirement for mouse DII4 in artery development. Genes \& development. 2004;18:2474-2478.

[107] Taylor KL, Henderson AM, Hughes CC. Notch activation during endothelial cell network formation in vitro targets the basic HLH transcription factor HESR-1 and downregulates VEGFR-2/KDR expression. Microvascular research. 2002;64:372-383.

[108] Holderfield MT, Henderson Anderson AM, Kokubo H, Chin MT, Johnson RL, Hughes CC. HESR1/CHF2 suppresses VEGFR2 transcription independent of binding to E-boxes. Biochemical and biophysical research communications. 2006;346:637-648.

[109] Davis RB, Curtis CD, Griffin CT. BRG1 promotes COUP-TFII expression and venous specification during embryonic vascular development. Development. 2013;140:1272-1281. 
[110] Henderson AM, Wang SJ, Taylor AC, Aitkenhead M, Hughes CC. The basic helix-loophelix transcription factor HESR1 regulates endothelial cell tube formation. The Journal of biological chemistry. 2001;276:6169-6176.

[111] Burns CE, Traver D, Mayhall E, Shepard JL, Zon LI. Hematopoietic stem cell fate is established by the Notch-Runx pathway. Genes \& development. 2005;19:2331-2342.

[112] Gering M, Patient R. Hedgehog signaling is required for adult blood stem cell formation in zebrafish embryos. Developmental cell. 2005;8:389-400.

[113] Kumano K, Chiba S, Kunisato A, et al. Notch1 but not Notch2 is essential for generating hematopoietic stem cells from endothelial cells. Immunity. 2003;18:699-711.

[114] Leung A, Ciau-Uitz A, Pinheiro P, et al. Uncoupling VEGFA functions in arteriogenesis and hematopoietic stem cell specification. Developmental cell. 2013;24:144-158.

[115] Richard C, Drevon C, Canto PY, et al. Endothelio-mesenchymal interaction controls runx1 expression and modulates the notch pathway to initiate aortic hematopoiesis.

Developmental cell. 2013;24:600-611.

[116] Lebestky T, Jung SH, Banerjee U. A Serrate-expressing signaling center controls Drosophila hematopoiesis. Genes \& development. 2003;17:348-353.

[117] Guiu J, Shimizu R, D'Altri T, et al. Hes repressors are essential regulators of hematopoietic stem cell development downstream of Notch signaling. The Journal of experimental medicine. 2013;210:71-84.

[118] Robert-Moreno A, Espinosa L, de la Pompa JL, Bigas A. RBPjkappa-dependent Notch function regulates Gata2 and is essential for the formation of intra-embryonic hematopoietic cells. Development. 2005;132:1117-1126.

[119] Robert-Moreno A, Guiu J, Ruiz-Herguido C, et al. Impaired embryonic haematopoiesis yet normal arterial development in the absence of the Notch ligand Jagged1. The EMBO journal. 2008;27:1886-1895.

[120] Clements WK, Kim AD, Ong KG, Moore JC, Lawson ND, Traver D. A somitic Wnt16/Notch pathway specifies haematopoietic stem cells. Nature. 2011;474:220-224.

[121] Clements WK, Traver D. Signalling pathways that control vertebrate haematopoietic stem cell specification. Nature reviews Immunology. 2013;13:336-348.

[122] Wilkinson RN, Pouget C, Gering M, et al. Hedgehog and Bmp polarize hematopoietic stem cell emergence in the zebrafish dorsal aorta. Developmental cell. 2009;16:909-916.

[123] Pardanaud L, Luton D, Prigent M, Bourcheix LM, Catala M, Dieterlen-Lievre F. Two distinct endothelial lineages in ontogeny, one of them related to hemopoiesis.

Development. 1996;122:1363-1371.

[124] Pouget C, Gautier R, Teillet MA, Jaffredo T. Somite-derived cells replace ventral aortic hemangioblasts and provide aortic smooth muscle cells of the trunk. Development. 2006;133:1013-1022.

[125] Sato $Y$, Watanabe $T$, Saito $D$, et al. Notch mediates the segmental specification of angioblasts in somites and their directed migration toward the dorsal aorta in avian embryos. Developmental cell. 2008;14:890-901.

[126] Kataoka H, Hayashi M, Kobayashi K, Ding G, Tanaka Y, Nishikawa SI. Region-specific Etv2 ablation revealed the critical origin of hemogenic capacity from Hox6-positive caudallateral primitive mesoderm. Experimental hematology. 2013. 
[127] Chong DC, Koo Y, Xu K, Fu S, Cleaver O. Stepwise arteriovenous fate acquisition during mammalian vasculogenesis. Developmental dynamics : an official publication of the American Association of Anatomists. 2011;240:2153-2165.

[128] Cleaver O, Krieg PA. VEGF mediates angioblast migration during development of the dorsal aorta in Xenopus. Development. 1998;125:3905-3914.

[129] Zhong TP, Childs S, Leu JP, Fishman MC. Gridlock signalling pathway fashions the first embryonic artery. Nature. 2001;414:216-220.

[130] Jin SW, Beis D, Mitchell T, Chen JN, Stainier DY. Cellular and molecular analyses of vascular tube and lumen formation in zebrafish. Development. 2005;132:5199-5209.

[131] Walmsley M, Ciau-Uitz A, Patient R. Adult and embryonic blood and endothelium derive from distinct precursor populations which are differentially programmed by BMP in Xenopus. Development. 2002;129:5683-5695.

[132] Kohli V, Schumacher JA, Desai SP, Rehn K, Sumanas S. Arterial and venous progenitors of the major axial vessels originate at distinct locations. Developmental cell. 2013;25:196-206.

[133] Nimmo R, Ciau-Uitz A, Ruiz-Herguido C, et al. MiR-142-3p controls the specification of definitive hemangioblasts during ontogeny. Developmental cell. 2013;26:237-249.

[134] Ciau-Uitz A, Wang L, Patient R, Liu F. ETS transcription factors in hematopoietic stem cell development. Blood cells, molecules \& diseases. 2013;51:248-255.

[135] Ciau-Uitz A, Pinheiro P, Gupta R, Enver T, Patient R. Tel1/ETV6 Specifies Blood Stem Cells through the Agency of VEGF Signaling. Developmental cell. 2010;18:569-578.

[136] Sabin FR. Studies on the origin of blood vessels and of red blood corpuscules as seen in the living blastoderm of chicks during the second day of incubation. Contrib Embryol. 1920;9:213-262.

[137] Murray PDF. The development in vitro of the blood of the early chick embryo. Proc Roy Soc, B. 1932;111:497-521.

[138] Mandal L, Banerjee U, Hartenstein V. Evidence for a fruit fly hemangioblast and similarities between lymph-gland hematopoiesis in fruit fly and mammal aorta-gonadalmesonephros mesoderm. Nature genetics. 2004;36:1019-1023.

[139] Stainier DY, Weinstein BM, Detrich HW, 3rd, Zon LI, Fishman MC. Cloche, an early acting zebrafish gene, is required by both the endothelial and hematopoietic lineages.

Development. 1995;121:3141-3150.

[140] Shalaby F, Ho J, Stanford WL, et al. A requirement for Flk1 in primitive and definitive hematopoiesis and vasculogenesis. Cell. 1997;89:981-990.

[141] Shalaby F, Rossant J, Yamaguchi TP, et al. Failure of blood-island formation and vasculogenesis in Flk-1-deficient mice. Nature. 1995;376:62-66.

[142] Lee D, Park C, Lee $H$, et al. ER71 acts downstream of BMP, Notch, and Wnt signaling in blood and vessel progenitor specification. Cell stem cell. 2008;2:497-507.

[143] Sumanas S, Gomez G, Zhao Y, Park C, Choi K, Lin S. Interplay among Etsrp/ER71, Scl, and Alk8 signaling controls endothelial and myeloid cell formation. Blood. 2008;111:45004510.

[144] Vogeli KM, Jin SW, Martin GR, Stainier DY. A common progenitor for haematopoietic and endothelial lineages in the zebrafish gastrula. Nature. 2006;443:337-339. 
[145] Weng W, Sukowati EW, Sheng G. On hemangioblasts in chicken. PloS one. 2007;2:e1228.

[146] Warga RM, Kane DA, Ho RK. Fate mapping embryonic blood in zebrafish: multi- and unipotential lineages are segregated at gastrulation. Developmental cell. 2009;16:744-755. [147] Kennedy M, D'Souza SL, Lynch-Kattman M, Schwantz S, Keller G. Development of the hemangioblast defines the onset of hematopoiesis in human ES cell differentiation cultures. Blood. 2007;109:2679-2687.

[148] Choi K, Kennedy M, Kazarov A, Papadimitriou JC, Keller G. A common precursor for hematopoietic and endothelial cells. Development. 1998;125:725-732.

[149] Huber TL, Kouskoff V, Fehling HJ, Palis J, Keller G. Haemangioblast commitment is initiated in the primitive streak of the mouse embryo. Nature. 2004;432:625-630.

[150] Ferkowicz MJ, Yoder MC. Blood island formation: longstanding observations and modern interpretations. Experimental hematology. 2005;33:1041-1047.

[151] Ueno H, Weissman IL. Clonal analysis of mouse development reveals a polyclonal origin for yolk sac blood islands. Developmental cell. 2006;11:519-533.

[152] Myers CT, Krieg PA. BMP-mediated specification of the erythroid lineage suppresses endothelial development in blood island precursors. Blood. 2013;122:3929-3939.

[153] Walmsley M, Cleaver D, Patient R. Fibroblast growth factor controls the timing of $\mathrm{Scl}$, Lmo2, and Runx1 expression during embryonic blood development. Blood.

2008;111:1157-1166.

[154] Fong GH, Zhang L, Bryce DM, Peng J. Increased hemangioblast commitment, not vascular disorganization, is the primary defect in flt-1 knock-out mice. Development. 1999;126:3015-3025.

[155] Gering M, Yamada Y, Rabbitts TH, Patient RK. Lmo2 and Scl/Tal1 convert non-axial mesoderm into haemangioblasts which differentiate into endothelial cells in the absence of Gata1. Development. 2003;130:6187-6199.

[156] Schneider VA, Mercola M. Wnt antagonism initiates cardiogenesis in Xenopus laevis. Genes \& development. 2001;15:304-315.

[157] Schoenebeck JJ, Keegan BR, Yelon D. Vessel and blood specification override cardiac potential in anterior mesoderm. Developmental cell. 2007;13:254-267.

[158] Simoes FC, Peterkin T, Patient R. Fgf differentially controls cross-antagonism between cardiac and haemangioblast regulators. Development. 2011;138:3235-3245.

[159] Kattman SJ, Huber TL, Keller GM. Multipotent flk-1+ cardiovascular progenitor cells give rise to the cardiomyocyte, endothelial, and vascular smooth muscle lineages.

Developmental cell. 2006;11:723-732.

[160] Peterkin T, Gibson A, Patient R. Common genetic control of haemangioblast and cardiac development in zebrafish. Development. 2009;136:1465-1474.

[161] Liu F, Kang I, Park C, et al. ER71 specifies Flk-1+ hemangiogenic mesoderm by inhibiting cardiac mesoderm and Wnt signaling. Blood. 2012;119:3295-3305.

[162] Palencia-Desai S, Kohli V, Kang J, Chi NC, Black BL, Sumanas S. Vascular endothelial and endocardial progenitors differentiate as cardiomyocytes in the absence of Etsrp/Etv2 function. Development. 2011;138:4721-4732.

[163] Van Handel B, Montel-Hagen A, Sasidharan R, et al. Scl represses cardiomyogenesis in prospective hemogenic endothelium and endocardium. Cell. 2012;150:590-605. 
[164] Parmar K, Mauch P, Vergilio JA, Sackstein R, Down JD. Distribution of hematopoietic stem cells in the bone marrow according to regional hypoxia. Proceedings of the National Academy of Sciences of the United States of America. 2007;104:5431-5436.

[165] Winkler IG, Barbier V, Wadley R, Zannettino AC, Williams S, Levesque JP. Positioning of bone marrow hematopoietic and stromal cells relative to blood flow in vivo: serially reconstituting hematopoietic stem cells reside in distinct nonperfused niches. Blood. 2010;116:375-385.

[166] Takubo K, Goda N, Yamada W, et al. Regulation of the HIF-1alpha level is essential for hematopoietic stem cells. Cell stem cell. 2010;7:391-402.

[167] Gerber HP, Malik AK, Solar GP, et al. VEGF regulates haematopoietic stem cell survival by an internal autocrine loop mechanism. Nature. 2002;417:954-958.

[168] Rehn M, Olsson A, Reckzeh K, et al. Hypoxic induction of vascular endothelial growth factor regulates murine hematopoietic stem cell function in the low-oxygenic niche. Blood. 2011;118:1534-1543.

[169] Imanirad P, Solaimani Kartalaei P, Crisan M, et al. HIF1alpha is a regulator of hematopoietic progenitor and stem cell development in hypoxic sites of the mouse embryo. Stem cell research. 2014;12:24-35.

[170] Harris JM, Esain V, Frechette GM, et al. Glucose metabolism impacts the spatiotemporal onset and magnitude of HSC induction in vivo. Blood. 2013;121:2483-2493.

[171] Simsek T, Kocabas F, Zheng J, et al. The distinct metabolic profile of hematopoietic stem cells reflects their location in a hypoxic niche. Cell stem cell. 2010;7:380-390.

[172] le Noble F, Moyon D, Pardanaud L, et al. Flow regulates arterial-venous differentiation in the chick embryo yolk sac. Development. 2004;131:361-375.

[173] Adamo L, Naveiras O, Wenzel PL, et al. Biomechanical forces promote embryonic haematopoiesis. Nature. 2009;459:1131-1135.

[174] North TE, Goessling W, Peeters M, et al. Hematopoietic stem cell development is dependent on blood flow. Cell. 2009;137:736-748.

[175] Wang L, Zhang P, Wei Y, Gao Y, Patient R, Liu F. A blood flow-dependent klf2a-NO signaling cascade is required for stabilization of hematopoietic stem cell programming in zebrafish embryos. Blood. 2011;118:4102-4110.

[176] Miller SB. Prostaglandins in health and disease: an overview. Seminars in arthritis and rheumatism. 2006;36:37-49.

[177] Feher I, Gidali J. Prostaglandin E2 as stimulator of haemopoietic stem cell proliferation. Nature. 1974;247:550-551.

[178] Gidali J, Feher I. The effect of E type prostaglandins on the proliferation of haemopoietic stem cells in vivo. Cell and tissue kinetics. 1977;10:365-373.

[179] Pelus LM. Association between colony forming units-granulocyte macrophage expression of la-like (HLA-DR) antigen and control of granulocyte and macrophage production. A new role for prostaglandin $\mathrm{E}$. The Journal of clinical investigation.

1982;70:568-578.

[180] Hoggatt J, Singh P, Sampath J, Pelus LM. Prostaglandin E2 enhances hematopoietic stem cell homing, survival, and proliferation. Blood. 2009;113:5444-5455.

[181] Cutler C, Multani P, Robbins D, et al. Prostaglandin-modulated umbilical cord blood hematopoietic stem cell transplantation. Blood. 2013;122:3074-3081. 
[182] Verma DS, Spitzer G, Zander AR, McCredie KB, Dicke KA. Prostaglandin E1-mediated augmentation of human granulocyte-macrophage progenitor cell growth in vitro. Leukemia research. 1981;5:65-71.

[183] Villablanca EJ, Pistocchi A, Court FA, et al. Abrogation of prostaglandin E2/EP4 signaling impairs the development of rag1+ lymphoid precursors in the thymus of zebrafish embryos. J Immunol. 2007;179:357-364.

[184] North TE, Goessling W, Walkley CR, et al. Prostaglandin E2 regulates vertebrate haematopoietic stem cell homeostasis. Nature. 2007;447:1007-1011.

[185] Goessling W, North TE, Loewer S, et al. Genetic interaction of PGE2 and Wnt signaling regulates developmental specification of stem cells and regeneration. Cell. 2009;136:1136-1147.

[186] Speth JM, Hoggatt J, Singh P, Pelus LM. Pharmacologic increase in HIF1alpha enhances hematopoietic stem and progenitor homing and engraftment. Blood.

2014;123:203-207.

[187] Safi R, Muramoto GG, Salter AB, et al. Pharmacological manipulation of the RAR/RXR signaling pathway maintains the repopulating capacity of hematopoietic stem cells in culture. Mol Endocrinol. 2009;23:188-201.

[188] Chanda B, Ditadi A, Iscove NN, Keller G. Retinoic acid signaling is essential for embryonic hematopoietic stem cell development. Cell. 2013;155:215-227.

[189] Linnemann AK, O'Geen H, Keles S, Farnham PJ, Bresnick EH. Genetic framework for GATA factor function in vascular biology. Proceedings of the National Academy of Sciences of the United States of America. 2011;108:13641-13646.

[190] Greenfest-Allen E, Malik J, Palis J, Stoeckert CJ, Jr. Stat and interferon genes identified by network analysis differentially regulate primitive and definitive erythropoiesis. BMC systems biology. 2013;7:38.

[191] Oostendorp RA, Harvey KN, Kusadasi N, et al. Stromal cell lines from mouse aortagonads-mesonephros subregions are potent supporters of hematopoietic stem cell activity. Blood. 2002;99:1183-1189.

[192] Oostendorp RA, Medvinsky AJ, Kusadasi N, et al. Embryonal subregion-derived stromal cell lines from novel temperature-sensitive SV40 T antigen transgenic mice support hematopoiesis. Journal of cell science. 2002;115:2099-2108.

[193] Taoudi S, Gonneau C, Moore K, et al. Extensive hematopoietic stem cell generation in the AGM region via maturation of VE-cadherin+CD45+ pre-definitive HSCs. Cell stem cell. 2008;3:99-108.

[194] Mirshekar-Syahkal B, Fitch SR, Ottersbach K. From greenhouse to garden: The changing soil of the hematopoietic stem cell microenvironment during development. Stem Cells. 2014.

[195] Mascarenhas MI, Parker A, Dzierzak E, Ottersbach K. Identification of novel regulators of hematopoietic stem cell development through refinement of stem cell localization and expression profiling. Blood. 2009;114:4645-4653.

[196] Magnusson M, Sierra MI, Sasidharan R, et al. Expansion on stromal cells preserves the undifferentiated state of human hematopoietic stem cells despite compromised reconstitution ability. PloS one. 2013;8:e53912. 
[197] Durand C, Robin C, Bollerot K, Baron MH, Ottersbach K, Dzierzak E. Embryonic stromal clones reveal developmental regulators of definitive hematopoietic stem cells. Proceedings of the National Academy of Sciences of the United States of America. 2007; 104:20838-20843.

[198] Mirshekar-Syahkal B, Haak E, Kimber GM, et al. Dlk1 is a negative regulator of emerging hematopoietic stem and progenitor cells. Haematologica. 2013;98:163-171.

[199] Peeters M, Ottersbach K, Bollerot K, et al. Ventral embryonic tissues and Hedgehog proteins induce early AGM hematopoietic stem cell development. Development.

2009;136:2613-2621.

[200] Fitch SR, Kimber GM, Wilson NK, et al. Signaling from the sympathetic nervous system regulates hematopoietic stem cell emergence during embryogenesis. Cell stem cell. 2012;11:554-566.

[201] Pandolfi PP, Roth ME, Karis A, et al. Targeted disruption of the GATA3 gene causes severe abnormalities in the nervous system and in fetal liver haematopoiesis. Nature genetics. 1995; 11:40-44.

[202] Jin H, Xu J, Wen Z. Migratory path of definitive hematopoietic stem/progenitor cells during zebrafish development. Blood. 2007;109:5208-5214.

[203] Dieterlen-Lievre F, Pouget C, Bollerot K, Jaffredo T. Are intra-aortic hemopoietic cells derived from endothelial cells during ontogeny? Trends in cardiovascular medicine.

2006;16:128-139.

[204] Grayfer L, Robert J. Colony-stimulating factor-1-responsive macrophage precursors reside in the amphibian (Xenopus laevis) bone marrow rather than the hematopoietic subcapsular liver. Journal of innate immunity. 2013;5:531-542.

[205] Bertrand JY, Kim AD, Teng S, Traver D. CD41+ cmyb+ precursors colonize the zebrafish pronephros by a novel migration route to initiate adult hematopoiesis.

Development. 2008;135:1853-1862.

[206] Nieuwkoop PD, Faber J. Normal Table of Xenopus laevis (Daudin). A systematic and chronological survey of the development from the fertilized egg till the end of metamorphosis. . Amsterdam: North-Holland Publishing Company.; 1967. 


\section{Figure Legends}

Figure 1. The ontogeny of embryonic blood and HSCs is conserved. The timing of hematopoietic activity is represented by shaded bars where the color gradient represents a peak in activity. Broken arrows represent migration and seeding of hematopoietic sites by hematopoietic stem or multipotent progenitor cells. Blood cells are produced in at least 3 distinct waves: a primitive wave, a pro-definitive wave and a definitive wave. In the first wave, blood islands in the yolk sac (YS, mouse), aVBI and pVBI (anterior and posterior Ventral Blood Islands, Xenopus) and anterior and posterior lateral mesoderm (ALM and PLM) give rise to erythroid (light blue) and myeloid cells (green) from extraembryonic (mouse) and lateral mesoderm (Xenopus and zebrafish). These populations will be gradually replaced by cells generated in the second and third waves.

The second (pro-definitive) wave comprises a transient population of multipotent erythromyeloid progenitors (EMPs, pink) which are thought to arise from hemogenic endothelium in the YS (mouse), posterior-lateral VBI (Xenopus) and posterior blood islands (PBI, zebrafish). In both mouse and frog there is evidence that EMPs go on to seed the fetal liver (FL, pink broken arrows) and give rise to erythroid and myeloid progeny $[15,16]$. The caudal hematopoietic tissue (CHT) is the FL equivalent in zebrafish $[26,202]$ and it develops from the PBI, which makes it difficult to detect migration and seeding of the CHT by EMPs. Notably, these EMPs can give rise to erythoid and myeloid progeny in vitro [40], but there is no evidence yet to suggest that zebrafish EMPs give rise to erythroid cells in vivo.

The third or definitive wave begins when HSCs arise in the aorta-gonad-mesonephros (AGM) region from hemogenic endothelium localised to the ventral wall of the main embryonic artery, the dorsal aorta (DA, dark blue bars). Generation of de novo HSCs from hemogenic endothelium is the termed endothelial to hematopoietic transition (EHT) and has been 
demonstrated in vivo in zebrafish [30, 66, 70] and ex-vivo in mouse embryos [67]. In most vertebrates (human, mouse, frog, chick), HSCs emerge into the lumen of the DA and form readily detectable clusters [203]. In zebrafish, EHT occurs not into the lumen of the DA but into the space between the DA and the posterior cardinal vein [26, 27, 66]. Soon after emergence, HSCs enter embryonic circulation and go on to seed the main embryonic hematopoietic site, the FL or its equivalent in zebrafish, the CHT. There they expand, differentiate into erythroid and myeloid but not lymphoid progenitors[1, 15, 26, 28, 29]. This is a transient site, and HSCs will eventually seed the final hematopoietic organs, the bone marrow (BM) in mouse, the FL and BM in frog and the kidney marrow in zebrafish (dark blue broken arrows)[27, 202]. In frog, the BM is thought to be a reservoir rather than a site for the production of mature blood cells [204]. The kidney marrow is seeded very early in zebrafish larvae (at around 4 days post fertilisation, dpf) but it is not thought to be the main hematopoietic organ until after the first week of development [26]. There is evidence suggesting that a small but significant population of HSCs can seed the kidney marrow without going through the CHT [205].

The embryonic staging for each of the model organisms is shown as days post-coitum (E) for mouse, Xenopus developmental stage according to Nieuwkoop and Faber [206] and in hours or days post-fertilisation (hpf or dpf) for zebrafish. For each of the organisms, key stages and/or sites of hematopoiesis are depicted above each of the timelines.

Figure2. Model for hemangioblast potential. In vivo hemangioblasts are subjected to developmental constraints (arrows) that prevent them to produce both blood and endothelial progeny and instead differentiate only in one of these lineages (A). When hemangiobalsts are removed from the embryo, dissociated and cultured in vitro, the developmental constraints 
imposed by the embryo are disrupted and, as a consequence, many more of them express their potential to produce both blood and endothelial progeny $(\mathbf{B})$.

\section{Figure 3. Cellular hierarchy from nascent mesoderm to HSC generation in the ventral}

wall of the DA. HSCs originate from lateral plate mesoderm expressing Flk1, these cells first give rise to hemangioblasts and subsequently endothelial cells that migrate to the midline to form the DA. After coalescing in the midline, the DA forms a lumen and gets polarised to produce hemogenic endothelium and HSCs in the ventral wall.

Figure 1

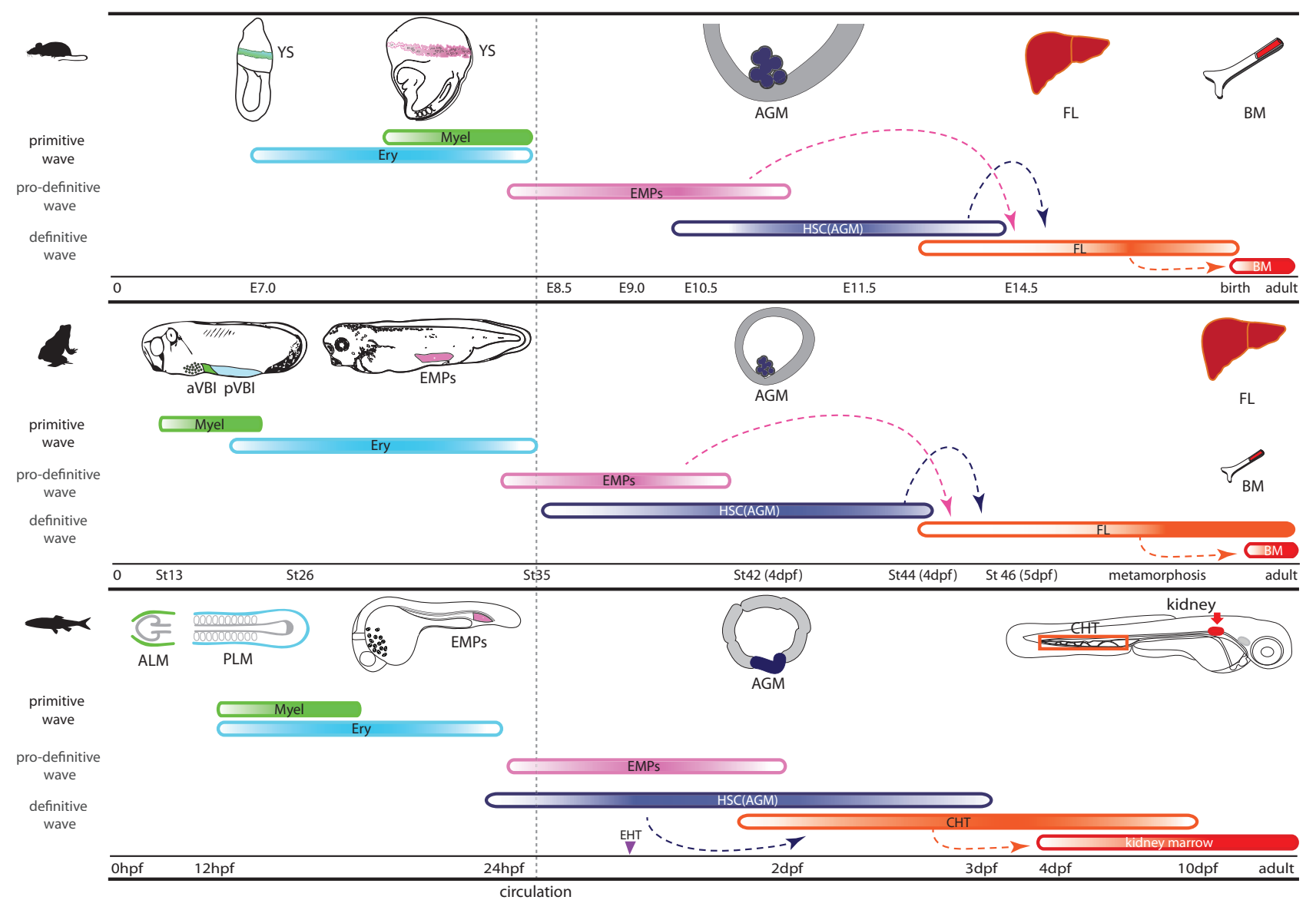


Figure 2

A

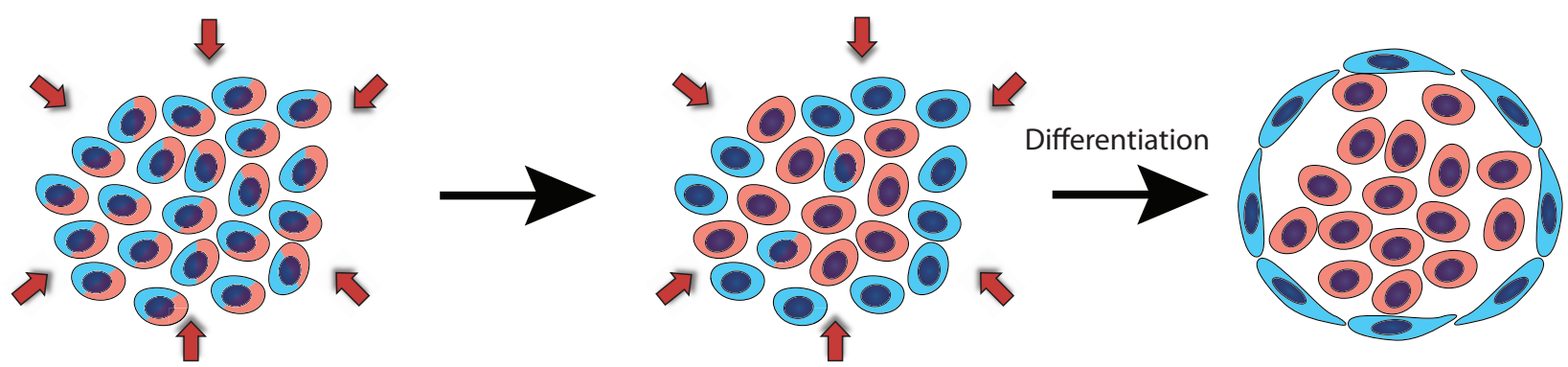

B

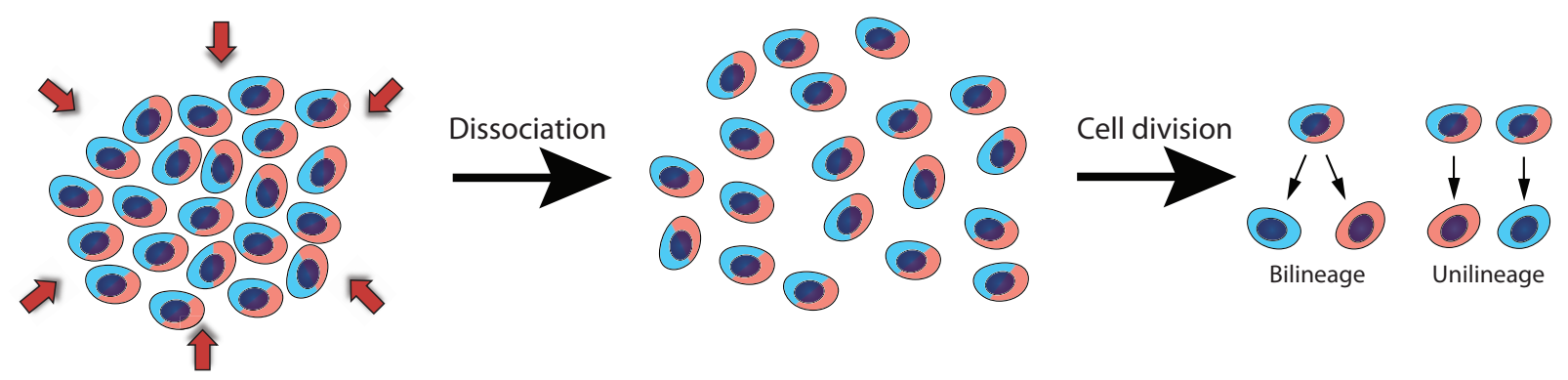

Legend

$\checkmark$ Developmental constraint Bilineage potential $\bigcirc($ Unilineage potential 
Figure 3

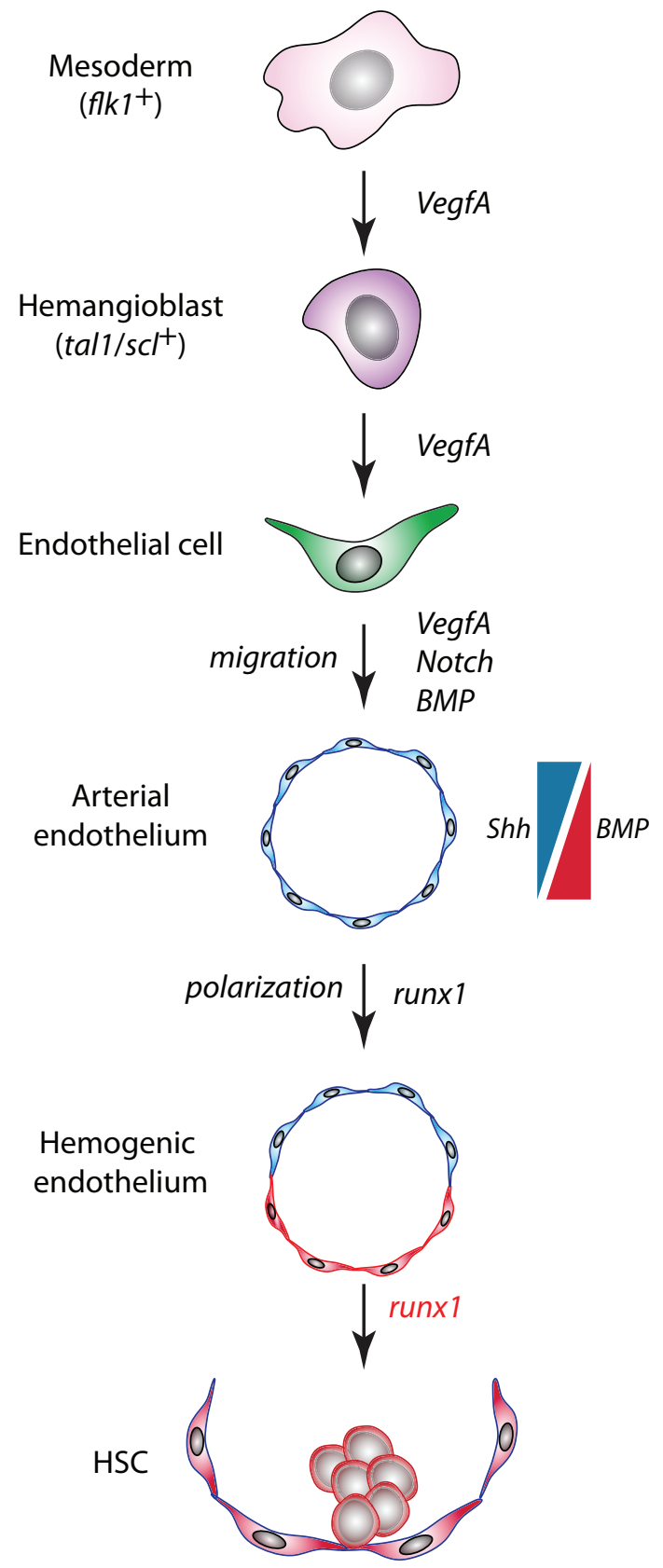

